DOI: http://dx.doi.org/10.21123/bsj.2020.17.3.0841

\title{
Modified Mathematical Model of Tumor Treatment by Radiotherapy
}

\author{
Saad Naji AL-Azzawi*
}

Fatima Ahmed Shihab

Department of Mathematics, College of Science for Women, University of Baghdad, Baghdad, Iraq.

*Corresponding author: saadnaji58@gmail.com*, afatima979@gmail.com

*ORCID ID: https://orcid.org/0000-0002-6865-9798*, https://orcid.org/0000-0002-5499-8262

Received 25/9/2018, Accepted 22/7/2019, Published 1/9/2020

This work is licensed under a Creative Commons Attribution 4.0 International License.

\begin{abstract}
:
In this research, a mathematical model of tumor treatment by radiotherapy is studied and a new modification for the model is proposed as well as introducing the check for the suggested modification. Also the stability of the modified model is analyzed in the last section.
\end{abstract}

\section{Mathematics Subject Classification:}

Key words: Cancer, Laplace Adomian Decomposition method,Radiotherapy.

\section{Introduction:}

One of the most important applications of differential equations in medicine is cancer growth and treatment. Cancer is nothing but an uncontrolled growth of abnormal cells inside the body (1). The origin of the word cancer is credited from the Greek physician Hippocrates (460-370 $\mathrm{BC})$, who is considered as "Father of Medicine". Hippocrates used the terms carcinos and carcinoma to describe non-ulcer forming and ulcer-forming tumors. In Greek, these words refer to a crab, most likely applied to the disease, because the fingerlike spreading projections from a cancer called to mind the shape of a crab (2). There are five main cancer groups, including, Leukemias, Sarcomas, Carcinomas, Lymphomas, and Brain tumors (3). According to the reports of the Cancer Research Institute, about 1,252,000 cases were diagnosed, with 547,000 deaths in 1995 in the United States alone (4). The International Agency for Research on Cancer reported that 12.7 million new cancer cases were detected in 2008. Today, there are new techniques for the detection of cancer and this will increase the chances of survival to more than $50 \%$. There are several treatment techniques which are used to treat cancer, such as surgery, chemotherapy, radiotherapy, immunotherapy, transplant one marrow and stem cells, hormone therapy, drug therapy, and clinical trials. The idea of using the qualitative theory of ordinary differential equations goes back to the twenties of the past century when Lotka and Volterra formulated a simple mathematical model in population dynamics theory (5). They described the interaction between the predator and the prey in a model called predator-prey model, which is a very important problem in ecology. In 1973, Bell proposed a mathematical model consisting of two equations based on the predator-prey model (6). De Boer and Hogeweg (1986) introduced a model consists of 10 ODEs and 3 additional equations describing several players of immune response. This model also covered all the phenomena from uncontrolled tumor growth to tumor regression due to immune system response (7). Kuznetsov (1994) presented a mathematical model of CTL (Cytotoxic T Lymphocytes i.e. cells with antitumor activity) cells response to the growth of immunogenic tumor, and he explained a number of phenomena, including sneaking through, dormant state of the tumor, and immunostimulation (8). Adam and Bellomo (1997) published a good summary on the tumor-immune dynamics, and it was based on Kuznetsov's work (1). Kirschner and Panetta (1998) described the dynamics between tumor cells, effector cells, and the cytokine interleukine-2 (IL-2) by a mathematical model, which is considered as a modulator of the immune stimulus (9). de Pillis and Radunskaya (2001 and 2006) proposed detailed models about the immune response differentiating between Natural Killer cells (NK-cells), CD8+ cytotoxic Tcells, and other lymphocytes (10)(11). 
In this paper, a mathematical model is reformulated with a new modification in one of its terms, specifically the radiation harvesting term. This term is responsible of the effect of radiation on the tumor cells. The new modification describes more powerful performance for the radiation without being affected by the change in parameters values. The value of the parameter in the new modification is calculated by using medical data of a patient and then the stability of the modified model is analyzed to explain the effect of the new term.

\section{The Mathematical Model}

In this section, we present the model that will be modified with new form to improve its performance in the treatment of tumors. The following model studies the coexistence of the healthy and cancer cells when treated with radiotherapy (12):

$$
\begin{aligned}
& \frac{d H}{d t}=\alpha_{1} H\left(1-\frac{H}{k_{1}}\right)-\beta_{1} H T-\varepsilon \gamma H \\
& \frac{d T}{d t}=\alpha_{2} T\left(1-\frac{T}{k_{2}}\right)-\beta_{2} H T-\gamma T \\
& {\left[n t_{0}, n t_{0}+l\right]}
\end{aligned}
$$

With initial conditions: $H(0)=h, T(0)=\tau$. Where $H$ is the concentration of healthy cells, $T$ is the concentration of tumor cells, $h, \tau$ are the initial concentrations of healthy cells and tumor cells respectively, $\alpha_{1}, \alpha_{2}>0$ are the growth rates of healthy cells and tumor cells respectively, $k_{1}, k_{2}>$ 0 are the carrying capacities of healthy cells and tumor cells respectively, $\beta_{1}, \beta_{2}$ are the respective competition coefficients of healthy cells and tumor cells respectively, $\varepsilon>0$ is the proportion coefficient of the radiation, and $\gamma$ is the strategy of radiation (12). Before we suggest the modifications of model (1), we have to check for the effect of the radiotherapy on the tumor cells in the tumor site (when $H=0$ ).

$\frac{d T}{d t}=\alpha_{2} T\left(1-\frac{T}{k_{2}}\right)-\gamma T$

Solving the above equation to find $T(t)$ :

$$
\begin{aligned}
& \frac{d T}{d t}=\left(\alpha_{2}-\gamma\right) T-\frac{\alpha_{2}}{k_{2}} T^{2} \\
& \int \frac{d T}{T\left(\left(\alpha_{2}-\gamma\right)-\frac{\alpha_{2}}{k_{2}} T\right)}=\int d t \\
& \frac{1}{\alpha_{2}-\gamma} \ln T-\frac{1}{\alpha_{2}-\gamma} \ln \left|\left(\alpha_{2}-\gamma\right)-\frac{\alpha_{2}}{k_{2}} T\right|=t+c
\end{aligned}
$$

$\ln \left|\frac{T}{\left(\alpha_{2}-\gamma\right)-\frac{\alpha_{2}}{k_{2}} T}\right|=\left(\alpha_{2}-\gamma\right) t+\left(\alpha_{2}-\gamma\right) c$, where $\left(\alpha_{2}-\gamma\right)-\frac{\alpha_{2}}{k_{2}} T \neq 0$

$\frac{T}{\left(\alpha_{2}-\gamma\right)-\frac{\alpha_{2}}{k_{2}} T}=k e^{\left(\alpha_{2}-\gamma\right) t}$, where $k=e^{\left(\alpha_{2}-\gamma\right) c}$

$T(t)=\frac{\left(\alpha_{2}-\gamma\right) k}{\frac{\alpha_{2} k}{k_{2}}+e^{-\left(\alpha_{2}-\gamma\right) t}}$

Now, we have two cases to discuss:

1) When $\alpha_{2}>\gamma$ then as $t \rightarrow \infty, T(t) \rightarrow \frac{k_{2}\left(\alpha_{2}-\gamma\right)}{\alpha_{2}}$

2) When $\alpha_{2}<\gamma$ then as $t \rightarrow \infty, T(t) \rightarrow 0$

From the cases above, we notice that (2) is affected by the values of $\alpha_{2}, \gamma$ which are the growth rate of tumor cells and the amount of radiation respectively. It is known that there is no cure that completely eliminates cancer but can only stop its growth. We need to think of the case of eliminating the tumor completely, so we have to find a modification which is not affected by any change in the parameters values.

\section{The Suggested Modification}

As mentioned before, the new modification is not affected by any change in parameters values. The radiation harvesting term $\gamma T$ in the second equation will be replaced by $\alpha_{3} \gamma T^{3}$ as follows:

$\frac{d H}{d t}=\alpha_{1} H\left(1-\frac{H}{k_{1}}\right)-\beta_{1} H T-\varepsilon \gamma H$

$\frac{d T}{d t}=\alpha_{2} T\left(1-\frac{T}{k_{2}}\right)-\beta_{2} H T-\alpha_{3} \gamma T^{3}$ ,$t \in$

$\left[n t_{0}, n t_{0}+l\right]$

Where $\alpha_{3}>0$

The new term decreases the rate of change in the tumor concentration and kills more of tumor cells than model (1). Now, we check for the effect of the radiotherapy on the tumor cells in the tumor site (when $h=0$, that is $H=0$ ):

$\frac{d T}{d t}=\alpha_{2} T\left(1-\frac{T}{k_{2}}\right)-\alpha_{3} \gamma T^{3}$

Solving the above equation to find $T(t)$ :

$T(t)=\frac{\frac{\alpha_{2}}{\alpha_{3} k_{2} \gamma} k-\sqrt{\left(\frac{\alpha_{2}^{2} k^{2}}{\alpha_{3}^{2} k_{2}^{2} \gamma^{2}}\right)-4 \frac{\alpha_{2}}{\alpha_{3} \gamma} k e^{-2 \alpha_{2} t}\left(1-k e^{2 \alpha_{2} t}\right)}}{2\left(e^{-2 \alpha_{2} t}-k\right)}$

We see that as $t \rightarrow \infty, T(t) \rightarrow 0$ and this explains that model (3) could be more powerful than model (1). 


\section{Laplace Adomian Decomposition Method}

Now, we introduce the Laplace Adomian decomposition method which will be used in the solution of model (3) when $H \neq 0$. This method is a combination of two methods Laplace transformation and the Adomian decomposition method, and it is used when we have nonlinear terms in the differential equation. The Adomian decomposition method simplifies the nonlinear terms before taking the Laplace transform, since Laplace transform deals with linear differential equations with constant coefficients. The Laplace Adomian decomposition method needs less work in comparison with the traditional Adomian decomposition method and it also decreases the calculations.

Consider the following system (13):

$$
\begin{aligned}
& L_{t} u_{1}=R_{1}\left(u_{1}, \ldots, u_{n}\right)+N_{1}\left(u_{1}, \ldots, u_{n}\right)+g_{1} \\
& L_{t} u_{2}=R_{2}\left(u_{1}, \ldots, u_{n}\right)+N_{2}\left(u_{1}, \ldots, u_{n}\right)+g_{2} \\
& \quad \vdots \\
& L_{t} u_{n}=R_{n}\left(u_{1}, \ldots, u_{n}\right)+N_{n}\left(u_{1}, \ldots, u_{n}\right)+g_{n}
\end{aligned}
$$

With the initial conditions

$u_{i}(0)=u_{i 0}, i=1, \ldots, n$

Where $L_{t}$ is a first-order differential operator, $R_{i}$ and $N_{i}, i=1, \ldots, n$ are linear and nonlinear operators, respectively, and $g_{i}, i=1, \ldots, n$ are analytic functions. Now, we apply Laplace transform to both sides of system (5) and use the properties of Laplace transform with the initial conditions (6), we get:

$$
\begin{aligned}
& \mathcal{L}\left[u_{1}\right]= \\
& \frac{1}{s}\left(u_{1}(0)+\mathcal{L}\left[g_{1}\right]\right)+\frac{1}{s} \mathcal{L}\left[R_{1}\left(u_{1}, \ldots, u_{n}\right)\right]+ \\
& \frac{1}{s} \mathcal{L}\left[N_{1}\left(u_{1}, \ldots, u_{n}\right)\right] \\
& \mathcal{L}\left[u_{2}\right]= \\
& \frac{1}{s}\left(u_{2}(0)+\mathcal{L}\left[g_{2}\right]\right)+\frac{1}{s} \mathcal{L}\left[R_{2}\left(u_{1}, \ldots, u_{n}\right)\right]+ \\
& \frac{1}{s} \mathcal{L}\left[N_{2}\left(u_{1}, \ldots, u_{n}\right)\right] \\
& \quad \quad \\
& \mathcal{L}\left[u_{n}\right]= \\
& \frac{1}{s}\left(u_{n}(0)+\mathcal{L}\left[g_{n}\right]\right)+\frac{1}{s} \mathcal{L}\left[R_{n}\left(u_{1}, \ldots, u_{n}\right)\right]+ \\
& \frac{1}{s} \mathcal{L}\left[N_{n}\left(u_{1}, \ldots, u_{n}\right)\right]
\end{aligned}
$$

Representing the solution as an infinite series, namely

$$
u_{i}(t)=\sum_{j=0}^{\infty} u_{i j}(t), \quad i=1, \ldots, n
$$

The terms $u_{i j}(t)$ are to be recursively computed. The nonlinear operator $N_{i}$ is decomposed as follows:

$N_{i}\left(u_{1}, \ldots, u_{n}\right)=\sum_{j=0}^{\infty} A_{i j}, i=1, \ldots, n$

And $A_{i j}$ are the so-called Adomian polynomials that can be derived for various classes of nonlinearity according to specific algorithms set by Adomian. We will expand few terms of the infinite series for one variable and two-variable Adomian polynomials as follows:

Let $A_{i j}=f\left(u_{i j}\right)$

$A_{i 0}=f\left(u_{i 0}\right)$

$A_{i 1}=u_{i 1} f^{\prime}\left(u_{i 0}\right)$

$\vdots$

And for the two-variable Adomian polynomials

$A=f(u, v)$, Where

$u=\sum_{j=0}^{\infty} u_{i j}, v=\sum_{j=0}^{\infty} v_{i j}, i$

$=1, \ldots, n$

$A_{i 0}=f\left(u_{i 0}, v_{i 0}\right)$

$A_{i 1}=\left.u_{i 1} \frac{\partial f}{\partial u_{i 0}}\right|_{\left(u_{i 0}, v_{i 0}\right)}+\left.v_{i 1} \frac{\partial f}{\partial v_{i 0}}\right|_{\left(u_{i 0}, v_{i 0}\right)}$

$\vdots$

Now, let

$\mu_{i}(s)=\frac{1}{s}\left(u_{i}(0)+\mathcal{L}\left[g_{i}\right]\right)$,

$i$

$=1, \ldots, n$

Substituting (8), (9) and (11) into (7), and using the linearity of Laplace transform, we get

$$
\begin{aligned}
& \sum_{j=0}^{\infty} \mathcal{L}\left[u_{1 j}\right]= \mu_{1}(s)+\frac{1}{s} \sum_{j=0}^{\infty} \mathcal{L}\left[R_{1}\left(u_{1 j}, \ldots, u_{n j}\right)\right] \\
&+\frac{1}{s} \sum_{j=0}^{\infty} \mathcal{L}\left[A_{1 j}\right] \\
& \sum_{j=0}^{\infty} \mathcal{L}\left[u_{2 j}\right]=\mu_{2}(s)+\frac{1}{s} \sum_{j=0}^{\infty} \mathcal{L}\left[R_{2}\left(u_{1 j}, \ldots, u_{n j}\right)\right] \\
&+\frac{1}{s} \sum_{j=0}^{\infty} \mathcal{L}\left[A_{2 j}\right]
\end{aligned}
$$




$$
\begin{aligned}
& \sum_{j=0}^{\infty} \mathcal{L}\left[u_{n j}\right] \\
& =\mu_{n}(s)+\frac{1}{s} \sum_{j=0}^{\infty} \mathcal{L}\left[R_{n}\left(u_{1 j}, \ldots, u_{n j}\right)\right] \\
& +\frac{1}{s} \sum_{j=0}^{\infty} \mathcal{L}\left[A_{n j}\right]
\end{aligned}
$$

Then we have the following recurrence relations from corresponding terms on both sides of (12):

$$
\begin{aligned}
& \mathcal{L}\left[u_{i 0}(t)\right]=\mu_{i}(s) \\
& \mathcal{L}\left[u_{i 1}(t)\right]=\frac{1}{s} \mathcal{L}\left[R_{i}\left(u_{10}, \ldots, u_{n 0}\right)\right]+\frac{1}{s} \mathcal{L}\left[A_{i 0}\right] \\
& \mathcal{L}\left[u_{i 2}(t)\right]=\frac{1}{s} \mathcal{L}\left[R_{i}\left(u_{11}, \ldots, u_{n 1}\right)\right]+\frac{1}{s} \mathcal{L}\left[A_{i 1}\right] \\
& \quad \vdots \\
& \mathcal{L}\left[u_{i(j+1)}(t)\right]=\frac{1}{s} \mathcal{L}\left[R_{i}\left(u_{1 j}, \ldots, u_{n j}\right)\right]+\frac{1}{s} \mathcal{L}\left[A_{i j}\right]
\end{aligned}
$$

Applying the inverse Laplace transform to the first equation in (13) gives the initial approximation:

$$
u_{i 0}(t)=\mathcal{L}^{-1}\left[\mu_{i}(s)\right], \quad i=1, \ldots, n
$$

Substituting these values of $u_{i 0}$ into the inverse Laplace transform of the second equation in (13) gives $u_{i 1}$

$u_{i 1}=\mathcal{L}^{-1}\left[\frac{1}{\mathrm{~s}} \mathcal{L}\left[R_{i}\left(u_{11}, \ldots, u_{n 1}\right)\right]+\frac{1}{\mathrm{~s}} \mathcal{L}\left[A_{i 1}\right]\right]$

The other terms $u_{i 2}, u_{i 3}, \ldots$ can be obtained recursively from the formula:

$$
\begin{aligned}
& u_{i(j+1)}(t)=\mathcal{L}^{-1}\left[\frac{1}{\mathrm{~s}} \mathcal{L}\left[R_{i}\left(u_{1 j}, \ldots, u_{n j}\right)\right]+\right. \\
& \left.\frac{1}{\mathrm{~s}} \mathcal{L}\left[A_{i j}\right]\right], j=1,2, \ldots
\end{aligned}
$$

\section{Solution of the Modified Model}

Now, we introduce the solution of model (3) by using Laplace Adomian decomposition method as follows:

$\frac{d H}{d t}=\left(\alpha_{1}-\varepsilon \gamma\right) H-\frac{\alpha_{1}}{k_{1}} H^{2}-\beta_{1} H T$

$\frac{d T}{d t}=\alpha_{2} T-\frac{\alpha_{2}}{k_{2}} T^{2}-\alpha_{3} \gamma T^{3}-\beta_{2} H T$

$\left[n t_{0}, n t_{0}+l\right]$

With initial conditions: $H(0)=h, T(0)=\tau$. Applying the Laplace transform to both sides of (17), we get

$$
\begin{aligned}
& s \mathcal{L}[H]-H(0)=\mathcal{L}\left[\left(\alpha_{1}-\varepsilon \gamma\right) H\right]-\mathcal{L}\left[\frac{\alpha_{1}}{k_{1}} H^{2}\right]- \\
& \mathcal{L}\left[\beta_{1} H T\right] \\
& s \mathcal{L}[T]-T(0)=\mathcal{L}\left[\alpha_{2} T\right]-\mathcal{L}\left[\frac{\alpha_{2}}{k_{2}} T^{2}\right]- \\
& \mathcal{L}\left[\alpha_{3} \gamma T^{3}\right]-\mathcal{L}\left[\beta_{2} H T\right]
\end{aligned}
$$

Applying the initial conditions and using the linearity property of Laplace transform to (18):

$\mathcal{L}[H]=\frac{h}{s}+\frac{\left(\alpha_{1}-\varepsilon \gamma\right)}{s} \mathcal{L}[H]-\frac{\alpha_{1}}{s k_{1}} \mathcal{L}\left[H^{2}\right]-$ $\frac{\beta_{1}}{s} \mathcal{L}[H T]$

$\stackrel{\mathcal{L}}{s}[T]=\frac{\tau}{s}+\frac{\alpha_{2}}{s} \mathcal{L}[T]-\frac{\alpha_{2}}{s k_{2}} \mathcal{L}\left[T^{2}\right]-\frac{\alpha_{3} \gamma}{s} \mathcal{L}\left[T^{3}\right]-$ $\frac{\beta_{2}}{s} \mathcal{L}[H T]$

Now, we represent the solution as an infinite series, namely,

$$
H=\sum_{n=0}^{\infty} H_{n}, \quad T=\sum_{n=0}^{\infty} T_{n}
$$

The terms $H_{n}$ and $T_{n}$ are to be recursively computed. Also the nonlinear terms in the system are represented as follows:

$$
A=H^{2}, B=H T, C=T^{2}, D=T^{3}
$$

The nonlinear operators $A, B, C$, and $D$ are decomposed as follows:

$$
\begin{aligned}
& A=\sum_{n=0}^{\infty} A_{n}, B=\sum_{n=0}^{\infty} B_{n}, C=\sum_{n=0}^{\infty} C_{n}, \text { and } D \\
& =\sum_{n=0}^{\infty} D_{n}
\end{aligned}
$$

Where $A_{n}, B_{n}, C_{n}$, and $D_{n}$ are the so-called Adomian polynomials and we will expand them as follows:

$$
\begin{array}{lcc}
A_{0}=H_{0}^{2} & B_{0}=H_{0} T_{0} \\
A_{1}=2 H_{1} H_{0} & , & B_{1}=H_{0} T_{1}+H_{1} T_{0} \\
A_{2}=2 H_{2} H_{0}+H_{1}^{2} & , & B_{2}=H_{0} T_{2}+H_{1} T_{1}+ \\
H_{2} T_{0} & &
\end{array}
$$

$$
\begin{array}{lrrr}
\quad & & \multicolumn{1}{c}{} \\
C_{0}=T_{0}^{2} & , & D_{0}=T_{0}^{3} \\
C_{1}=2 T_{1} T_{0} & D_{1}=3 T_{1} T_{0}^{2} \\
C_{2}=2 T_{2} T_{0}+T_{1}^{2} & , & D_{2}=3 T_{2} T_{0}^{2}+ \\
3 T_{0} T_{1}^{2} & &
\end{array}
$$

Substituting (20) and (22) into (19): 
$\mathcal{L}\left[\sum_{n=0}^{\infty} H_{n}\right]=\frac{h}{s}+\frac{\left(\alpha_{1}-\varepsilon \gamma\right)}{s} \mathcal{L}\left[\sum_{n=0}^{\infty} H_{n}\right]-$

$\frac{\alpha_{1}}{s k_{1}} \mathcal{L}\left[\sum_{n=0}^{\infty} A_{n}\right]-\frac{\beta_{1}}{s} \mathcal{L}\left[\sum_{n=0}^{\infty} B_{n}\right]$

$\mathcal{L}\left[\sum_{n=0}^{\infty} T_{n}\right]=$

$\frac{\tau}{s}+\frac{\alpha_{2}}{s} \mathcal{L}\left[\sum_{n=0}^{\infty} T_{n}\right]-\frac{\alpha_{2}}{s k_{2}} \mathcal{L}\left[\sum_{n=0}^{\infty} C_{n}\right]-$

$\frac{\alpha_{3} \gamma}{s} \mathcal{L}\left[\sum_{n=0}^{\infty} D_{n}\right]-\frac{\beta_{2}}{s} \mathcal{L}\left[\sum_{n=0}^{\infty} B_{n}\right]$

Matching both sides of (23) yields the following iterative algorithms:

$$
\begin{aligned}
& \mathcal{L}\left[H_{0}\right]=\frac{h}{s} \\
& \mathcal{L}\left[H_{1}\right]=\frac{\left(\alpha_{1}-\varepsilon \gamma\right)}{s} \mathcal{L}\left[H_{0}\right]-\frac{\alpha_{1}}{s k_{1}} \mathcal{L}\left[A_{0}\right]-\frac{\beta_{1}}{s} \mathcal{L}\left[B_{0}\right] \\
& \quad \vdots \\
& \mathcal{L}\left[H_{n+1}\right]=\frac{\left(\alpha_{1}-\varepsilon \gamma\right)}{s} \mathcal{L}\left[H_{n}\right]-\frac{\alpha_{1}}{s k_{1}} \mathcal{L}\left[A_{n}\right]-\frac{\beta_{1}}{s} \mathcal{L}\left[B_{n}\right]
\end{aligned}
$$

And

$$
\begin{aligned}
& \mathcal{L}\left[T_{0}\right]=\frac{\tau}{s} \\
& \mathcal{L}\left[T_{1}\right]=\frac{\alpha_{2}}{s} \mathcal{L}\left[T_{0}\right]-\frac{\alpha_{2}}{s k_{2}} \mathcal{L}\left[C_{0}\right]-\frac{\alpha_{3} \gamma}{s} \mathcal{L}\left[D_{0}\right]- \\
& \frac{\beta_{2}}{s} \mathcal{L}\left[B_{0}\right] \\
& \quad \vdots \\
& \mathcal{L}\left[T_{n+1}\right]=\frac{\alpha_{2}}{s} \mathcal{L}\left[T_{n}\right]-\frac{\alpha_{2}}{s k_{2}} \mathcal{L}\left[C_{n}\right]-\frac{\alpha_{3} \gamma}{s} \mathcal{L}\left[D_{n}\right]- \\
& \frac{\beta_{2}}{s} \mathcal{L}\left[B_{n}\right]
\end{aligned}
$$

Taking the first term of each series:

$$
\mathcal{L}\left[H_{0}\right]=\frac{h}{s}, \mathcal{L}\left[T_{0}\right]=\frac{\tau}{s}
$$

Applying the inverse Laplace transform to (26), we get

$$
H_{0}=h, \quad T_{0}=\tau
$$

Using the values from (27) to obtain $H_{1}, T_{1}$

$$
\begin{gathered}
\mathcal{L}\left[H_{1}\right]=\frac{\left(\alpha_{1}-\varepsilon \gamma\right)}{s} \mathcal{L}[h]-\frac{\alpha_{1}}{s k_{1}} \mathcal{L}\left[h^{2}\right]-\frac{\beta_{1}}{s} \mathcal{L}[h \tau] \\
=\left(\alpha_{1}-\varepsilon \gamma-\frac{\alpha_{1} h}{k_{1}}-\beta_{1} \tau\right) \frac{h}{s^{2}} \\
\mathcal{L}\left[T_{1}\right]=\frac{\alpha_{2}}{s} \mathcal{L}[\tau]-\frac{\alpha_{2}}{s k_{2}} \mathcal{L}\left[\tau^{2}\right]-\frac{\alpha_{3} \gamma}{s} \mathcal{L}\left[\tau^{3}\right]- \\
\frac{\beta_{2}}{s} \mathcal{L}[h \tau]=\left(\alpha_{2}-\beta_{2} h-\frac{\alpha_{2} \tau}{k_{2}}-\alpha_{3} \gamma \tau^{2}\right) \frac{\tau}{s^{2}}
\end{gathered}
$$

Applying the inverse Laplace transform to (28), we get

$H_{1}=\left(\alpha_{1}-\varepsilon \gamma-\frac{\alpha_{1} h}{k_{1}}-\beta_{1} \tau\right) h t$

$T_{1}=\left(\alpha_{2}-\beta_{2} h-\frac{\alpha_{2} \tau}{k_{2}}-\alpha_{3} \gamma \tau^{2}\right) \tau t$

Using the values from (29) to obtain $H_{2}, T_{2}$ with the same procedure:

$$
\begin{aligned}
& H_{2}=\left[( \alpha _ { 1 } - \varepsilon \gamma - \frac { 2 \alpha _ { 1 } h } { k _ { 1 } } - \beta _ { 1 } \tau ) \left(\alpha_{1}-\varepsilon \gamma-\frac{\alpha_{1} h}{k_{1}}-\right.\right. \\
& \left.\left.\beta_{1} \tau\right)-\beta_{1} \tau\left(\alpha_{2}-\beta_{2} h-\frac{\alpha_{2} \tau}{k_{2}}-\alpha_{3} \gamma \tau^{2}\right)\right] h \frac{t^{2}}{2}
\end{aligned}
$$

$$
\begin{aligned}
& T_{2}=\left[( \alpha _ { 2 } - \beta _ { 2 } h - \frac { 2 \alpha _ { 2 } \tau } { k _ { 2 } } - 3 \alpha _ { 3 } \gamma \tau ^ { 2 } ) \left(\alpha_{2}-\beta_{2} h-\right.\right. \\
& \left.\left.\frac{\alpha_{2} \tau}{k_{2}}-\alpha_{3} \gamma \tau^{2}\right)-\beta_{2} h\left(\alpha_{1}-\varepsilon \gamma-\frac{\alpha_{1} h}{k_{1}}-\beta_{1} \tau\right)\right] \tau \frac{t^{2}}{2}
\end{aligned}
$$

\begin{tabular}{|c|c|c|c|}
\hline Parameter & Description & Value & Source \\
\hline$\alpha_{1}$ & $\begin{array}{l}\text { The growth rate of } \\
\text { healthy cells }\end{array}$ & 0.2 & (12) \\
\hline$\alpha_{2}$ & $\begin{array}{l}\text { The growth rate of } \\
\text { tumor cells }\end{array}$ & 0.45 & (12) \\
\hline$k_{1}$ & $\begin{array}{l}\text { The carrying } \\
\text { capacity of healthy } \\
\text { cells }\end{array}$ & 0.65 & (12) \\
\hline$k_{2}$ & $\begin{array}{l}\text { The carrying } \\
\text { capacity of tumor } \\
\text { cells }\end{array}$ & 1 & (12) \\
\hline$\varepsilon$ & $\begin{array}{l}\text { The proportion } \\
\text { coefficient of } \\
\text { radiation }\end{array}$ & 0.3 & (12) \\
\hline$\gamma$ & $\begin{array}{l}\text { The strategy of } \\
\text { radiation }\end{array}$ & 0.65 & (12) \\
\hline$\beta_{1}$ & $\begin{array}{l}\text { The respective } \\
\text { competition } \\
\text { coefficient of } \\
\text { healthy cells }\end{array}$ & 0.5 & (12) \\
\hline$\beta_{2}$ & $\begin{array}{l}\text { The respective } \\
\text { competition } \\
\text { coefficient of tumor } \\
\text { cells }\end{array}$ & 0.55 & (12) \\
\hline
\end{tabular}

Because of the uniform convergence property, few terms of each series of $H$ and $T$ are enough for a good accuracy. Then we can write the solution of system (3) as follows:

$$
\begin{aligned}
& H(t)=h+\left(\alpha_{1}-\varepsilon \gamma-\frac{\alpha_{1} h}{k_{1}}-\beta_{1} \tau\right) h t+\left[\left(\alpha_{1}-\right.\right. \\
& \left.\varepsilon \gamma-\frac{2 \alpha_{1} h}{k_{1}}-\beta_{1} \tau\right)\left(\alpha_{1}-\varepsilon \gamma-\frac{\alpha_{1} h}{k_{1}}-\beta_{1} \tau\right)- \\
& \left.\beta_{1} \tau\left(\alpha_{2}-\beta_{2} h-\frac{\alpha_{2} \tau}{k_{2}}-\alpha_{3} \gamma \tau^{2}\right)\right] h \frac{t^{2}}{2} \\
& T(t)=\tau+\left(\alpha_{2}-\beta_{2} h-\frac{\alpha_{2} \tau}{k_{2}}-\alpha_{3} \gamma \tau^{2}\right) \tau t+\left[\left(\alpha_{2}-\right.\right. \\
& \left.\beta_{2} h-\frac{2 \alpha_{2} \tau}{k_{2}}-3 \alpha_{3} \gamma \tau^{2}\right)\left(\alpha_{2}-\beta_{2} h-\frac{\alpha_{2} \tau}{k_{2}}-\alpha_{3} \gamma \tau^{2}\right) \\
& \left.\quad-\beta_{2} h\left(\alpha_{1}-\varepsilon \gamma-\frac{\alpha_{1} h}{k_{1}}-\beta_{1} \tau\right)\right] \tau \frac{t^{2}}{2}
\end{aligned}
$$

\section{Calculation of the Unknown Parameter $\left(\alpha_{3}\right)$}

In this section, a full study of the stability of model (3) will be analyzed to explain the behavior of the model with the new modification. First, we have to calculate the value of the unknown parameter $\left(\alpha_{3}\right)$ in the suggested modification. In table 1 medical data of a patient suffering from renal cell carcinoma (RCC) during radiotherapy will be used to calculate $\left(\alpha_{3}\right)$.

Table 1. Values and Description of the Parameters in Model (3). 
Now, we introduce medical data of a cancer patient suffering from (RCC) during the treatment with radiotherapy. The data includes the time of radiation dose (in hours) and the concentration of tumor cells corresponding to each time as in table 2.

Table 2. Medical Data of a Cancer Patient During Radiotherapy.

\begin{tabular}{ll}
\hline Time (hours) & Concentration of tumor cells \\
\hline 0 & 0.8 \\
1 & 0.69 \\
2.25 & 0.20 \\
\hline
\end{tabular}

The initial conditions (12) are:

$$
\begin{aligned}
H(0) & =h=0.5 \\
T(0) & =\tau=0.8
\end{aligned}
$$

Substituting the values of the parameters in Table 1 and the initial conditions from (33) into (32), we get $T(t)$ :

$$
\begin{aligned}
& T(t)=0.8-\left(0.148+0.3328 \alpha_{3}\right) t+ \\
& \left(0.16613376 \alpha_{3}^{2}+0.146432 \alpha_{3}-\right. \\
& 0.028109076) t^{2}
\end{aligned}
$$

When $t=1$ and $T=0.69$ in (34), we obtain

$$
\begin{aligned}
& 0.16613376 \alpha_{3}^{2}-0.186368 \alpha_{3}- \\
& 0.066109076=0
\end{aligned}
$$

$$
\begin{aligned}
\left(\alpha_{3}\right)_{1,2}= & \frac{0.186368 \pm \sqrt{0.078664828}}{0.33226752} \\
& =\left\{\begin{array}{c}
1.405013978 \\
-0.283219106
\end{array}\right.
\end{aligned}
$$

Then $\alpha_{3}=1.405013978>0$

After finding the value of $\alpha_{3}$; then we can study the stability of model (3) and the linearization theorem will be used for this purpose as follows:

$\frac{d H}{d t}=\alpha_{1} H\left(1-\frac{H}{k_{1}}\right)-\beta_{1} H T-\varepsilon \gamma H$

$\frac{d T}{d t}=\alpha_{2} T\left(1-\frac{T}{k_{2}}\right)-\beta_{2} H T-\alpha_{3} \gamma T^{3}$

Setting $\left(\frac{d H}{d t}=0, \frac{d T}{d t}=0\right)$, we get:

$\alpha_{1} H\left(1-\frac{H}{k_{1}}\right)-\beta_{1} H T-\varepsilon \gamma H=0$

$\alpha_{2} T\left(1-\frac{T}{k_{2}}\right)-\beta_{2} H T-\alpha_{3} \gamma T^{3}=0$

From the first equation, we have: $H=0$ or $T=$ $\frac{1}{\beta_{1}}\left(\alpha_{1}-\varepsilon \gamma-\frac{\alpha_{1}}{k_{1}} H\right)$

When $H=0$ in the second equation, we get: $T=0$ or $\left(\alpha_{3} \gamma T^{2}+\frac{\alpha_{2}}{k_{2}} T-\alpha_{2}\right)=0$
Then we have the following two equilibrium points:

$E_{1}=(0,0)$ and $E_{2}=\left(0, \frac{-\alpha_{2}+\sqrt{\alpha_{2}^{2}+4 \alpha_{3} \alpha_{2} \gamma k_{2}^{2}}}{2 \alpha_{3} \gamma k_{2}}\right)$

When $T=\frac{1}{\beta_{1}}\left(\alpha_{1}-\varepsilon \gamma-\frac{\alpha_{1}}{k_{1}} H\right)$ in the second equation, we get the following cubic equation:

$\frac{\alpha_{1}^{3} \alpha_{3} \gamma}{k_{1}^{3} \beta_{1}^{3}} H^{3}+\left(\frac{\alpha_{1} \beta_{2}}{k_{1} \beta_{1}}-\frac{\alpha_{1}^{2} \alpha_{2}}{\beta_{1}^{2} k_{1}^{2} k_{2}}-\frac{3 \alpha_{1}^{2} \alpha_{3} \gamma}{k_{1}^{2} \beta_{1}^{3}}\left(\alpha_{1}-\right.\right.$

$\varepsilon \gamma)) H^{2}+\left(-\frac{\alpha_{1} \alpha_{2}}{k_{1} \beta_{1}}-\frac{\beta_{2}}{\beta_{1}}\left(\alpha_{1}-\varepsilon \gamma\right)+\frac{2 \alpha_{1} \alpha_{2}}{\beta_{1}^{2} k_{1} k_{2}}\left(\alpha_{1}-\right.\right.$

$\varepsilon \gamma)$

$\left.+\frac{3 \alpha_{1} \alpha_{3} \gamma}{k_{1} \beta_{1}^{3}}\left(\alpha_{1}-\varepsilon \gamma\right)^{2}\right) H+\left(\frac{\alpha_{2}}{\beta_{1}}\left(\alpha_{1}-\varepsilon \gamma\right)-\right.$

$\left.\frac{\alpha_{2}}{\beta_{1}^{2} k_{2}}\left(\alpha_{1}-\varepsilon \gamma\right)^{2}-\frac{\alpha_{3} \gamma}{\beta_{1}^{3}}\left(\alpha_{1}-\varepsilon \gamma\right)^{3}\right)=0$

Substituting the values of the parameters into (36) and (37), we get:

$E_{1}=(0,0)$

$E_{2}=(0,0.497564521)$

And equation (37) will be:

$H^{3}+0.740832892 H^{2}-1.300170734 H+$

$0.020927857=0$

(38)

The roots of (38) are:

$H_{1}=0.81777$

$H_{2}=0.01625$

$H_{3}=-1.57485$

We take $H_{2}$ only since $H_{1}$ gives negative value for

$T$. Then we have the third equilibrium point:

$E_{3}=\left(0.01625,2.25 \times 10^{-11}\right)$

The linearization matrix of system (3) is

$A=$
$\left[\begin{array}{cc}\alpha_{1}-\varepsilon \gamma-\frac{2 \alpha_{1}}{k_{1}} \mathrm{H}-\beta_{1} \mathrm{~T} & -\beta_{1} \mathrm{H} \\ -\beta_{2} \mathrm{~T} & \alpha_{2}-\frac{2 \alpha_{2}}{k_{2}} \mathrm{~T}-\beta_{2} \mathrm{H}-3 \alpha_{3} \gamma \mathrm{T}^{2}\end{array}\right]$

Now, we calculate $A$ at each equilibrium point

1) $A_{\mathrm{E}_{1}}=\left[\begin{array}{cc}0.005 & 0 \\ 0 & 0.45\end{array}\right]$

The eigenvalues are

$\lambda_{1}=0.45, \lambda_{2}=0.005$

We notice that $E_{1}$ is unstable equilibrium point which is the case when there are no healthy cells and tumor cells.

2) $A_{\mathrm{E}_{2}}=\left[\begin{array}{cc}-0.24378226 & 0 \\ -0.273660486 & -0.676095964\end{array}\right]$

The eigenvalues are

$\lambda_{1}=-0.24378226, \lambda_{2}=-0.676095964$

Since both of the eigenvalues are negative then $E_{2}$ is an asymptotically stable equilibrium point. This 
means that when there are only tumor cells, then the radiation will eliminate them successfully.

3)

$A_{\mathrm{E}_{3}}=$

$\left[\begin{array}{cc}5.000000011 \times 10^{-3} & -8.125 \times 10^{-3} \\ -1.2375 \times 10^{-11} & -3.93750002 \times 10^{-3}\end{array}\right]$

$p(\lambda)=\lambda^{2}-1.062499991 \times 10^{-3} \lambda-$

$1.02515625 \times 10^{-13}=0$

The eigenvalues are

$\lambda_{1}=0.0010625, \lambda_{2}=-9.648528 \times 10^{-11}$

Since the eigenvalues have opposite signs, then $E_{3}$ is an unstable saddle node. In this case there are healthy and tumor cells in the tumor site and this causes a delay in the work of radiotherapy which gives a chance to tumor cells to grow and divide.

\section{Conclusion:}

In this paper, we presented a dynamical system of two equations which describe the coexistence between healthy cells and tumor cells. A modification was introduced to enhance the performance of the radiotherapy by suggesting a new harvesting term $\alpha_{3} \gamma T^{3}$ which assumes that the radiation kills more than one tumor cell in the same time without increasing the dose of radiation $\gamma$. The Laplace Adomian decomposition method was used for solving the system and to find $\alpha_{3}$, we used medical data of a cancer patient during the treatment with radiotherapy. Finally, we studied the stability of the modified model when all the parameters are known.

\section{Authors' declaration:}

- Conflicts of Interest: None.

- We hereby confirm that all the Figures and Tables in the manuscript are mine ours. Besides, the Figures and images, which are not mine ours, have been given the permission for re-publication attached with the manuscript.

- The author has signed an animal welfare statement.

- Ethical Clearance: The project was approved by the local ethical committee in University of Baghdad.

\section{References:}

1. Adam JA, Bellomo N. A Survey of Models for Tumor-Immune System Dynamics. Boston, USA: Birkhauser Series on Modeling and Simulation in Science, Engineering and Technology [internet], Birkhauser.1997. Available from:

https://doi.org/10.1002/10970258(20001130)19:22\%3C3140::AIDSIM610\%3E3.0.CO;2-Q
2. American Cancer Society, Early History of Cancer; [cited $2017 \mathrm{Feb} 2]$.

Available from:

https://www.cancer.org/cancer/cancer.../history...canc er/what-is-cancer

3. Cancer Research UK, What is cancer; [cited 2017 Feb 2]. Available from: https://www.cancerresearchuk.org/aboutcancer/what-is-cancer

4. Chang W, Crowl L, Malm E, Todd-Brown K, Thomas L, Vrable M. Analyzing Immunotherapy and Chemotherapy of Tumors through Mathematical Modeling. HMC [internet]. 2003 May [cited 2017 Feb 19]. Available from:

https://www.math.utah.edu/ crowl/research/tumor

5. Tsygvintsev A, Marino S, Kirschner DE. Mathematical Methods and Models in Biomedicine [internet]. Berlin, Germany: Springer; 2013. A Mathematical Model of Gene Therapy for the Treatment of Cancer [cited 2017 Jan 26]. p. 355-73. Available from:

https://www.researchgate.net/publication/267829423

6. Pang L, Shen L, Zhao Z. Mathematical Modeling and Analysis of Tumor Treatment Regimens with Pulsed Immunotherapy and Chemotherapy. Comput Math Methods Med [internet]. 2016 [cited 2017 Jan 26]; 2016(2016):1-12. Available from: http://dx.doi.org/10.1155/2016/6260474

7. Roesch K, Hasenclever D, Scholz M. Modeling Lymphoma Therapy and Outcome. Bull Math Biol [internet]. 2014 Jan [cited 2017 Jan 26];76(2):40130. Available from:

https://link.springer.com/article/10.1007/s11538-0139925-3

8. Kuznetsov VA, Makalkin IA, Taylor MA, Perelson AS. Nonlinear Dynamics of Immunogenic Tumors: Parameter Estimation and Global Bifurcation Analysis. Bull Math Biol [internet]. 1994 [cited 2017 Jan 26];56(2): 295-21. Available from:

https://www.sciencedirect.com/science/article/pii/S00 92824005802605

9. Kirschner D, Panetta JC. Modeling Immunotherapy of the Tumor-Immune Interaction. J Math Biol [internet]. 1998 [cited 2017 Feb 19];37(3):235-52. Available from: https://doi.org/10.1007/s002850050127

10. De Pillis L, Radunskaya A. A Mathematical Tumor Model with Immune Resistance and Drug Therapy: An Optimal Control Approach, J Theor Med [internet]. 2001 [cited 2017 Jan 26];3(2):79-100. http://dx.doi.org/10.1080/10273660108833067

11. De Pillis L, Gu W, Radunskaya A. Mixed Immunotherapy and Chemotherapy of Tumors: Modeling, Applications and Biological Interpretations. J Theor Biol [internet]. $2006 \mathrm{Mar}$ [cited 2017 Jan 26]; 238(4): 841-62. Available from: https://doi.org/10.1016/j.jtbi.2005.06.037

12. Liu Z, Yang C. A Mathematical Model of Cancer Treatment by Radiotherapy. Comput Math Methods Med [internet]. 2014 Nov [cited 2017 May 22];2014(2014):1-12. Available from: 
http://dx.doi.org/10.1155/2014/172923

13. Radunskaya A, Kim R, Woods II T. (2018), Mathematical Modeling of Tumor Immune Interactions: A Closer Look at the Role of a PD-L1 Inhibitor in Cancer Immunotherapy. Spora [internet]. 2018 [cited 2018 Dec 15];4(1):25-41. Available from:

http://doi.org/10.30707/SPORA4.1Radunskaya

14. Arrowsmith DK., Place CM. Dynamical Systems: Differential Equations, Maps and Chaotic Behavior. London: Chapman and Hall; 1992. 330.

15. Hayder MA, Andrew JA, John W. (2018), Dynamical Density-Functional-Theory-Based Modeling of Tissue Dynamics: Application to Tumor Growth. Phys Rev E [internet]. 2018 Aug [cited 2018 Oct 10];98(2-1): 022407. Available from:

https://doi.org/10.1103/PhysRevE.98.022407

16. Medina MA. Mathematical Modeling of Cancer Metabolism. Crit Rev Oncol Hematol [internet]. 2018 Apr [cited 2018 Oct 10];124(2018):37-40. Available from:

https://doi.org/10.1016/j.critrevonc.2018.02.004
17. Milad Shamsi, Mohsen Saghafian, Morteza Dejam and Amir Sanati-Nezhad. Mathematical Modeling of the Function of Warburg Effect in Tumor Microbiology. Sci Rep [internet]. 2018 Jun [cited 2018 Oct 10];8(1):8903. Available from: https://doi.org/10.1038/s41598-018-27303-6

18. Olivia M. A mathematical model of cancer networks with radiation therapy. J Young Investig [internet]. 2014 [cited 2017 Jun 13];27(6):17-26. Available from:

https://static1.squarespace.com/static/5443d7c7e4b06 e8b47de9a55/t/58dc7ecd6a4963e5f5c2c169/149084 5398292/JYI Dec2014 17to26-1.pdf

19. Sabir W, Mohammed AK. Approximation Algorithm for a System of Pantograph Equations. J Appl Math [internet]. 2012 Mar [cited 2017 Jan 26];2012(2012):1-9. Available from: http://dx.doi.org/10.1155/2012/714681

20. Sebastian B, Clare L, Dominique B, Lynn H, Philip H. Mathematical Modeling of Tumor-Tumor Distant Interactions Supports A Systemic Control of Tumor Growth. Am J Cancer [internet]. 2017 Jul [cited 2018 Oct 10];77(18):5183-5193. Available from: https://doi.org/10.1158/0008-5472.can-17-0564

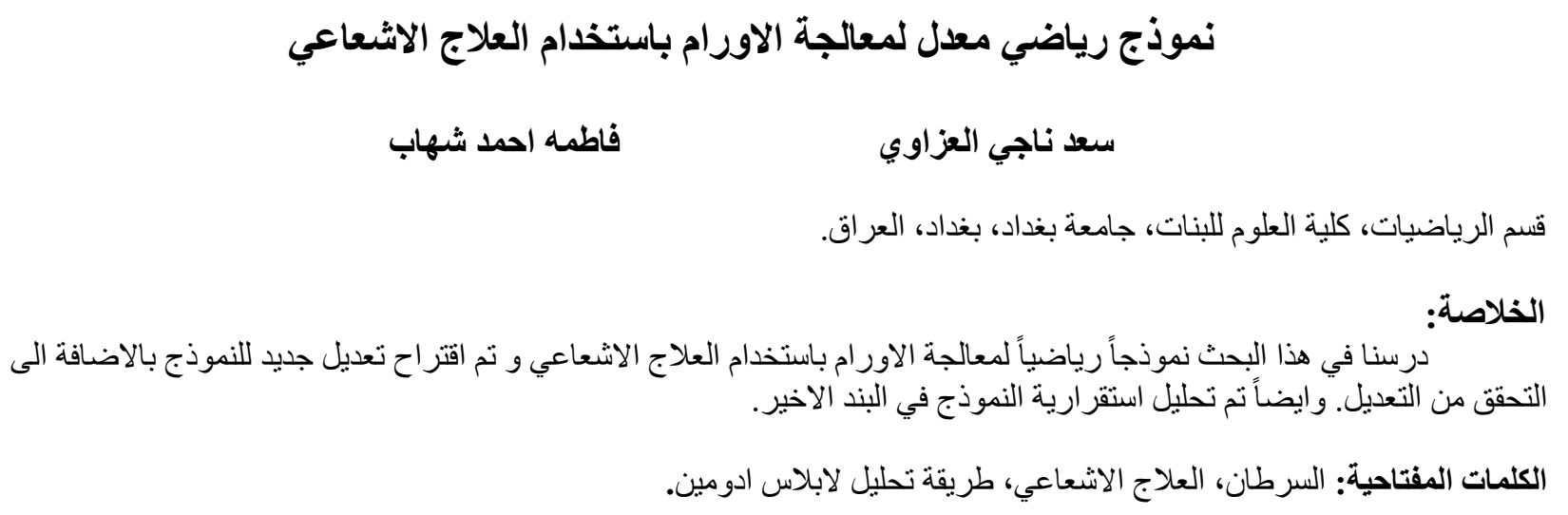

